\title{
Fuzzy Almost Strongly (r, s)-Semicontinuous Mappings
}

\author{
Seok Jong Lee ${ }^{1, *}$ and Jin Tae Kim² \\ Department of Mathematics, Chungbuk National University, Cheongju 361-763, Korea
}

\begin{abstract}
In this paper, we introduce the concept of fuzzy almost strongly $(r, s)$-semicontinuous mappings on intuitionistic fuzzy topological spaces in Šostak's sense. The relationships among fuzzy strongly $(r, s)$-semicontinuous, fuzzy almost $(r, s)$ continuous, fuzzy almost $(r, s)$-semicontinuous, and fuzzy almost strongly $(r, s)$-semicontinuous mappings are discussed. The characterization for the fuzzy almost strongly $(r, s)$-semicontinuous mappings is obtained.
\end{abstract}

Key Words: fuzzy continuous, fuzzy topology, fuzzy almost strongly $(r, s)$-semicontinuous

\section{Introduction}

The concept of fuzzy set was introduced by Zadeh [1]. Chang [2] defined fuzzy topological spaces. These spaces and its generalizations are later studied by several authors, one of which, developed by Šostak [3], used the idea of degree of openness. This type of generalization of fuzzy topological spaces was later rephrased by Chattopadhyay and his colleagues [4], and by Ramadan [5].

As a generalization of fuzzy sets, the concept of intuitionistic fuzzy sets was introduced by Atanassov [6] and Çoker [7] introduced intuitionistic fuzzy topological spaces using intuitionistic fuzzy sets. Using the idea of degree of openness and degree of nonopenness, Çoker and Demirci [8] defined intuitionistic fuzzy topological spaces in Šostak's sense as a generalization of smooth topological spaces and intuitionistic fuzzy topological spaces. Shi Zhong Bai [9] introduced the concept of fuzzy almost strongly semicontinuous mappings on Chang's fuzzy topological spaces.

In this paper, we introduce the concept of fuzzy almost strongly $(r, s)$-semicontinuous mappings on intuitionistic fuzzy topological spaces in Šstak's sense. The relationships among fuzzy strongly $(r, s)$ semicontinuous, fuzzy almost $(r, s)$-continuous, fuzzy almost $(r, s)$-semicontinuous, and fuzzy almost strongly $(r, s)$-semicontinuous mappings are discussed. The characterization for the fuzzy almost strongly $(r, s)$ semicontinuous mappings is obtained.

Manuscript received Apr. 25, 2012; revised Jun. 7, 2012; accepted Jun. $10,2012$.

*Corresponding author: Seok Jong Lee(sj1@chungbuk.ac.kr)

(c)The Korean Institute of Intelligent Systems. All rights reserved.

\section{Preliminaries}

For the nonstandard definitions and notations we refer to $[10,11,12,13]$.

Let $I(X)$ be a family of all intuitionistic fuzzy sets in $X$ and let $I \otimes I$ be the set of the pair $(r, s)$ such that $r, s \in I$ and $r+s \leq 1$

Definition 2.1. ([8]) Let $X$ be a nonempty set. An intuitionistic fuzzy topology in Šostak's sense(SoIFT for short) $\mathcal{T}=\left(\mathcal{T}_{1}, \mathcal{T}_{2}\right)$ on $X$ is a mapping $\mathcal{T}: I(X) \rightarrow I \otimes I$ which satisfies the following properties:

(1) $\mathcal{T}_{1}(\underline{0})=\mathcal{T}_{1}(\underline{1})=1$ and $\mathcal{T}_{2}(\underline{0})=\mathcal{T}_{2}(\underline{1})=0$.

(2) $\mathcal{T}_{1}(A \cap B) \geq \mathcal{T}_{1}(A) \wedge \mathcal{T}_{1}(B)$ and $\mathcal{T}_{2}(A \cap B) \leq \mathcal{T}_{2}(A) \vee$ $\mathcal{T}_{2}(B)$.

(3) $\mathcal{T}_{1}\left(\bigcup A_{i}\right) \geq \bigwedge \mathcal{T}_{1}\left(A_{i}\right)$ and $\mathcal{T}_{2}\left(\bigcup A_{i}\right) \leq \bigvee \mathcal{T}_{2}\left(A_{i}\right)$.

The $(X, \mathcal{T})=\left(X, \mathcal{T}_{1}, \mathcal{T}_{2}\right)$ is said to be an intuitionistic fuzzy topological space in Šostak's sense(SoIFTS for short). Also, we call $\mathcal{T}_{1}(A)$ a gradation of openness of $A$ and $\mathcal{T}_{2}(A)$ a gradation of nonopenness of $A$.

Definition 2.2. ([10, 11, 13]) Let $A$ be an intuitionistic fuzzy set in a $\operatorname{SoIFTS}\left(X, \mathcal{T}_{1}, \mathcal{T}_{2}\right)$ and $(r, s) \in I \otimes I$. Then $A$ is said to be

(1) fuzzy $(r, s)$-semiopen if $\operatorname{cl}(\operatorname{int}(A, r, s), r, s) \supseteq A$,

(2) fuzzy $(r, s)$-semiclosed if $\operatorname{int}(\operatorname{cl}(A, r, s), r, s) \subseteq A$,

(3) fuzzy $(r, s)$-regular open if $\operatorname{int}(\operatorname{cl}(A, r, s), r, s)=A$,

(4) fuzzy $(r, s)$-regular closed if $\operatorname{cl}(\operatorname{int}(A, r, s), r, s)=A$,

(5) fuzzy strongly $(r, s)$-semiopen if $A \subseteq \operatorname{int}(\operatorname{cl}(\operatorname{int}(A, r, s), r, s), r, s)$, 
(6) fuzzy strongly $(r, s)$-semiclosed if $A \supseteq \operatorname{cl}(\operatorname{int}(\operatorname{cl}(A, r, s), r, s), r, s)$.

Definition 2.3. ([13]) Let $\left(X, \mathcal{T}_{1}, \mathcal{T}_{2}\right)$ be a SoIFTS. For each $(r, s) \in I \otimes I$ and for each $A \in I(X)$, the fuzzy strongly $(r, s)$-semiinterior is defined by

$$
\operatorname{ssint}(A, r, s)=\bigcup\{B \in I(X) \mid B \subseteq A,
$$

$B$ is fuzzy strongly $(r, s)$-semiopen $\}$

and the fuzzy strongly $(r, s)$-semiclosure is defined by

$$
\operatorname{sscl}(A, r, s)=\bigcap\{B \in I(X) \mid A \subseteq B,
$$

$B$ is fuzzy strongly $(r, s)$-semiclosed $\}$.

Theorem 2.4. ([11]) (1) The fuzzy $(r, s)$-closure of a fuzzy $(r, s)$-open set is fuzzy $(r, s)$-regular closed for each $(r, s) \in I \otimes I$.

(2) The fuzzy $(r, s)$-interior of a fuzzy $(r, s)$-closed set is fuzzy $(r, s)$-regular open for each $(r, s) \in I \otimes I$.

Definition 2.5. ([11, 12, 14]) Let $f:\left(X, \mathcal{T}_{1}, \mathcal{T}_{2}\right) \rightarrow$ $\left(Y, \mathcal{U}_{1}, \mathcal{U}_{2}\right)$ be a mapping from a SoIFTS $X$ to a SoIFTS $Y$ and $(r, s) \in I \otimes I$. Then $f$ is called

(1) a fuzzy strongly $(r, s)$-semicontinuous mapping if $f^{-1}(B)$ is a fuzzy strongly $(r, s)$-semiopen set in $X$ for each fuzzy $(r, s)$-open set $B$ in $Y$,

(2) a fuzzy almost $(r, s)$-continuous mapping if $f^{-1}(B)$ is a fuzzy $(r, s)$-open set in $X$ for each fuzzy $(r, s)$ regular open set $B$ in $Y$,

(3) a fuzzy almost $(r, s)$-semicontinuous mapping if $f^{-1}(B)$ is a fuzzy $(r, s)$-semiopen set in $X$ for each fuzzy $(r, s)$-regular open set $B$ in $Y$.

Definition 2.6. ([11]) Let $x_{(\alpha, \beta)}$ be an intuitionistic fuzzy point in a SoIFTS $\left(X, \mathcal{T}_{1}, \mathcal{T}_{2}\right)$ and $(r, s) \in I \otimes I$. Then an intuitionistic fuzzy set $A$ in $X$ is called a fuzzy $(r, s)$ neighborhood of $x_{(\alpha, \beta)}$ if there is a fuzzy $(r, s)$-open set $B$ in $X$ such that $x_{(\alpha, \beta)} \in B \subseteq A$.

Definition 2.7. ([9]) Let $f:\left(X_{1}, \delta_{1}\right) \rightarrow\left(X_{2}, \delta_{2}\right)$ be a mapping from a fuzzy space $X_{1}$ to another fuzzy space $X_{2}$. Then $f$ is called a fuzzy almost strongly semicontinuous mapping if $f^{-1}(B)$ is a fuzzy strongly semiopen set of $X_{1}$ for each fuzzy regular open set $B$ of $X_{2}$.

\section{Fuzzy almost strongly $(r, s)$-semicontinuous mappings}

Now, we define the notion of fuzzy almost strongly $(r, s)$-semicontinuous mappings on intuitionistic fuzzy topological spaces in Šostak's sense, and then we investigate some of their properties.

Definition 3.1. Let $f:\left(X, \mathcal{T}_{1}, \mathcal{T}_{2}\right) \rightarrow\left(Y, \mathcal{U}_{1}, \mathcal{U}_{2}\right)$ be a mapping from a SoIFTS $X$ to a SoIFTS $Y$ and $(r, s) \in$ $I \otimes I$. Then $f$ is called a fuzzy almost strongly $(r, s)$ semicontinuous mapping if $f^{-1}(B)$ is a fuzzy strongly $(r, s)$-semiopen set in $X$ for each fuzzy $(r, s)$-regular open set $B$ in $Y$.

Definition 3.2. Let $f:\left(X, \mathcal{T}_{1}, \mathcal{T}_{2}\right) \rightarrow\left(Y, \mathcal{U}_{1}, \mathcal{U}_{2}\right)$ be a mapping from a SoIFTS $X$ to a SoIFTS $Y$ and $(r, s) \in$ $I \otimes I$. Then $f$ is said to be fuzzy almost strongly $(r, s)$ semicontinuous at an intuitionistic fuzzy point $x_{(\alpha, \beta)}$ in $X$ if for each fuzzy $(r, s)$-regular open set $B$ in $Y$ with $f\left(x_{(\alpha, \beta)}\right) \in B$, there is a fuzzy strongly $(r, s)$-semiopen set $A$ in $X$ such that $x_{(\alpha, \beta)} \in A$ and $f(A) \subseteq B$.

Theorem 3.3. Let $f:\left(X, \mathcal{T}_{1}, \mathcal{T}_{2}\right) \rightarrow\left(Y, \mathcal{U}_{1}, \mathcal{U}_{2}\right)$ be a mapping from a SoIFTS $X$ to a SoIFTS $Y$ and $(r, s) \in I \otimes I$. Then $f$ is fuzzy almost strongly $(r, s)$ semicontinuous if and only if $f$ is fuzzy almost strongly $(r, s)$-semicontinuous at each intuitionistic fuzzy point $x_{(\alpha, \beta)}$ in $X$.

Proof. Let $f$ be fuzzy almost strongly $(r, s)$ semicontinuous, $x_{(\alpha, \beta)}$ an intuitionistic fuzzy point in $X$, and $B$ a fuzzy $(r, s)$-regular open set in $Y$ with $f\left(x_{(\alpha, \beta)}\right) \in B$. Since $f$ is fuzzy almost strongly $(r, s)$-semicontinuous, $f^{-1}(B)$ is a fuzzy strongly $(r, s)$ semiopen set in $X$. Putting $A=f^{-1}(B)$. Then $A$ is fuzzy strongly $(r, s)$-semiopen in $X, x_{(\alpha, \beta)} \in A$, and $f(A)=f\left(f^{-1}(B)\right) \subseteq B$. Since $x_{(\alpha, \beta)}$ is an arbitrary intuitionistic fuzzy point in $X$, we conclude that $f$ is fuzzy almost strongly $(r, s)$-semicontinuous at each intuitionistic fuzzy point $x_{(\alpha, \beta)}$ in $X$.

Conversely, let $B$ be a fuzzy $(r, s)$-regular open set in $Y$ and $x_{(\alpha, \beta)} \in f^{-1}(B)$. Then $f\left(x_{(\alpha, \beta)}\right) \in B$. From the assumption, there is a fuzzy strongly $(r, s)$-semiopen set $A_{x_{(\alpha, \beta)}}$ in $X$ such that $x_{(\alpha, \beta)} \in A_{x_{(\alpha, \beta)}}$ and $f\left(A_{x_{(\alpha, \beta)}}\right) \subseteq$ $B$. Thus

$$
\begin{aligned}
f^{-1}(B) & =\bigcup\left\{x_{(\alpha, \beta)} \mid x_{(\alpha, \beta)} \in f^{-1}(B)\right\} \\
& \subseteq \bigcup\left\{A_{x_{(\alpha, \beta)}} \mid x_{(\alpha, \beta)} \in f^{-1}(B)\right\} \\
& \subseteq f^{-1}(B) .
\end{aligned}
$$

Hence $f^{-1}(B)=\bigcup\left\{A_{x_{(\alpha, \beta)}} \mid x_{(\alpha, \beta)} \in f^{-1}(B)\right\}$, which is a fuzzy strongly $(r, s)$-semiopen set in $X$. Therefore $f$ is fuzzy almost strongly $(r, s)$-semicontinuous. 
Remark 3.4. It is clear that the following implications are true:

(1) fuzzy strongly $(r, s)$-semicontinuous $\Rightarrow$ fuzzy almost strongly $(r, s)$-semicontinuous.

(2) fuzzy almost $(r, s)$-continuous $\Rightarrow$ fuzzy almost strongly $(r, s)$-semicontinuous.

(3) fuzzy almost strongly $(r, s)$-semicontinuous $\Rightarrow$ fuzzy almost $(r, s)$-semicontinuous.

However, the following examples show that all of the converses need not be true.

Example 3.5. Let $X=\{x, y, z\}$ and let $A_{1}, A_{2}, A_{3}$, and $A_{4}$ be intuitionistic fuzzy sets in $X$ defined as

$A_{1}(x)=(0.1,0.8), \quad A_{1}(y)=(0,1), \quad A_{1}(z)=(0.2,0.6)$

$A_{2}(x)=(0.5,0.5), A_{2}(y)=(0.5,0.5), A_{2}(z)=(0.5,0.5) ; A_{2}(x)=(0,0.7), A_{2}(y)=(0.2,0.7), A_{2}(z)=(0.2,0.8)$. $A_{3}(x)=(0.2,0.7), A_{3}(y)=(0.3,0.6), A_{3}(z)=(0.4,0.5)$ and

$A_{4}(x)=(0.6,0.3), A_{4}(y)=(0.5,0.5), A_{4}(z)=(0.7,0.1)$.

$A_{1}(x)=(0,0.5), A_{1}(y)=(0.3,0.5), A_{1}(z)=(0.3,0.5) ;$

Define $\mathcal{T}: I(X) \rightarrow I \otimes I$ and $\mathcal{U}: I(X) \rightarrow I \otimes I$ by $\mathcal{T}(A)=\left(\mathcal{T}_{1}(A), \mathcal{T}_{2}(A)\right)= \begin{cases}(1,0) & \text { if } A=\underline{0}, \underline{1} \\ \left(\frac{1}{2}, \frac{1}{3}\right) & \text { if } A=A_{1}, A_{2}, \\ (0,1) & \text { otherwise }\end{cases}$ and

$\mathcal{U}(A)=\left(\mathcal{U}_{1}(A), \mathcal{U}_{2}(A)\right)= \begin{cases}(1,0) & \text { if } A=\underline{0}, \underline{1} \\ \left(\frac{1}{2}, \frac{1}{3}\right) & \text { if } A=A_{3}, A_{4} \\ (0,1) & \text { otherwise }\end{cases}$

Then clearly $\mathcal{T}$ and $\mathcal{U}$ are SoIFTs on $X$. Consider a mapping $f:(X, \mathcal{T}) \rightarrow(X, \mathcal{U})$ defined by $f(x)=x, f(y)=y$, and $f(z)=z$. Note that

$$
\begin{gathered}
\operatorname{int}\left(\operatorname{cl}\left(A_{3}, \frac{1}{2}, \frac{1}{3}\right), \frac{1}{2}, \frac{1}{3}\right)=A_{3}, \\
\operatorname{int}\left(\operatorname{cl}\left(A_{4}, \frac{1}{2}, \frac{1}{3}\right), \frac{1}{2}, \frac{1}{3}\right)=\underline{1} \neq A_{2} \text { in }(X, \mathcal{U}) .
\end{gathered}
$$

Thus $A_{3}$ is fuzzy $\left(\frac{1}{2}, \frac{1}{3}\right)$-regular open but $A_{4}$ is not a fuzzy $\left(\frac{1}{2}, \frac{1}{3}\right)$-regular open set in $(X, \mathcal{U})$. Since

$$
\begin{aligned}
f^{-1}\left(A_{3}\right)=A_{3} & \subseteq \operatorname{int}\left(\operatorname{cl}\left(\operatorname{int}\left(A_{3}, \frac{1}{2}, \frac{1}{3}\right), \frac{1}{2}, \frac{1}{3}\right), \frac{1}{2}, \frac{1}{3}\right) \\
& =\operatorname{int}\left(\operatorname{cl}\left(A_{1}, \frac{1}{2}, \frac{1}{3}\right), \frac{1}{2}, \frac{1}{3}\right) \\
& =\operatorname{int}\left(A_{2}^{c}, \frac{1}{2}, \frac{1}{3}\right)=A_{2}
\end{aligned}
$$
Define $\mathcal{T}: I(X) \rightarrow I \otimes I$ and $\mathcal{U}: I(X) \rightarrow I \otimes I$ by

Example 3.6. Let $X=\{x, y, z\}$ and let $A_{1}$ and $A_{2}$ be intuitionistic fuzzy sets in $X$ defined as

and

we conclude that $f$ is fuzzy almost strongly $\left(\frac{1}{2}, \frac{1}{3}\right)$ semicontinuous. However, $f$ is neither fuzzy almost $\left(\frac{1}{2}, \frac{1}{3}\right)$-continuous nor fuzzy strongly $\left(\frac{1}{2}, \frac{1}{3}\right)$ semicontinuous. For $f^{-1}\left(A_{3}\right)=A_{3}$ is not fuzzy $\left(\frac{1}{2}, \frac{1}{3}\right)$-open in $(X, \mathcal{T})$ and

$$
\begin{aligned}
f^{-1}\left(A_{4}\right)=A_{4} & \nsubseteq \operatorname{int}\left(\operatorname{cl}\left(\operatorname{int}\left(A_{4}, \frac{1}{2}, \frac{1}{3}\right), \frac{1}{2}, \frac{1}{3}\right), \frac{1}{2}, \frac{1}{3}\right) \\
& =\operatorname{int}\left(\operatorname{cl}\left(A_{2}, \frac{1}{2}, \frac{1}{3}\right), \frac{1}{2}, \frac{1}{3}\right) \\
& =\operatorname{int}\left(A_{2}^{c}, \frac{1}{2}, \frac{1}{3}\right)=A_{2} .
\end{aligned}
$$

$$
\mathcal{T}(A)=\left(\mathcal{T}_{1}(A), \mathcal{T}_{2}(A)\right)= \begin{cases}(1,0) & \text { if } A=\underline{0}, \underline{1} \\ \left(\frac{1}{2}, \frac{1}{3}\right) & \text { if } A=A_{2} \\ (0,1) & \text { otherwise }\end{cases}
$$

and

$$
\mathcal{U}(A)=\left(\mathcal{U}_{1}(A), \mathcal{U}_{2}(A)\right)= \begin{cases}(1,0) & \text { if } A=\underline{0}, \underline{1} \\ \left(\frac{1}{2}, \frac{1}{3}\right) & \text { if } A=A_{1} \\ (0,1) & \text { otherwise }\end{cases}
$$

Then clearly $\mathcal{T}$ and $\mathcal{U}$ are SoIFTs on $X$. Consider a mapping $f:(X, \mathcal{T}) \rightarrow(X, \mathcal{U})$ defined by $f(x)=x, f(y)=y$, and $f(z)=z$. Note that

$$
\operatorname{int}\left(\operatorname{cl}\left(A_{1}, \frac{1}{2}, \frac{1}{3}\right), \frac{1}{2}, \frac{1}{3}\right)=A_{1} \text { in }(X, \mathcal{U}),
$$

and hence $A_{1}$ is fuzzy $\left(\frac{1}{2}, \frac{1}{3}\right)$-regular open in $(X, \mathcal{U})$. Since $f^{-1}\left(A_{1}\right)=A_{1}$ is fuzzy $\left(\frac{1}{2}, \frac{1}{3}\right)$-semiopen in $(X, \mathcal{T}), f$ is fuzzy almost $\left(\frac{1}{2}, \frac{1}{3}\right)$-semicontinuous. However, $f$ is not a fuzzy almost strongly $\left(\frac{1}{2}, \frac{1}{3}\right)$-semicontinuous mapping. For

$$
\begin{aligned}
f^{-1}\left(A_{1}\right)=A_{1} & \nsubseteq \operatorname{int}\left(\operatorname{cl}\left(\operatorname{int}\left(A_{1}, \frac{1}{2}, \frac{1}{3}\right), \frac{1}{2}, \frac{1}{3}\right), \frac{1}{2}, \frac{1}{3}\right) \\
& =\operatorname{int}\left(\operatorname{cl}\left(A_{2}, \frac{1}{2}, \frac{1}{3}\right), \frac{1}{2}, \frac{1}{3}\right) \\
& =\operatorname{int}\left(A_{2}^{c}, \frac{1}{2}, \frac{1}{3}\right)=A_{2} .
\end{aligned}
$$

Theorem 3.7. Let $f:\left(X, \mathcal{T}_{1}, \mathcal{T}_{2}\right) \rightarrow\left(Y, \mathcal{U}_{1}, \mathcal{U}_{2}\right)$ be a mapping from a SoIFTS $X$ to a SoIFTS $Y$ and $(r, s) \in I \otimes I$. Then the following statements are equivalent:

(1) $f$ is fuzzy almost strongly $(r, s)$-semicontinuous. 
International Journal of Fuzzy Logic and Intelligent Systems, vol. 12, no. 2, June 2012

(2) $f^{-1}(B)$ is fuzzy strongly $(r, s)$-semiclosed in $X$ for each fuzzy $(r, s)$-regular closed set $B$ in $Y$.

(3) For each fuzzy $(r, s)$-closed set $B$ in $Y$,

$$
\operatorname{sscl}\left(f^{-1}(\operatorname{cl}(\operatorname{int}(B, r, s), r, s)), r, s\right) \subseteq f^{-1}(B) .
$$

(4) For each fuzzy $(r, s)$-open set $B$ in $Y$,

$$
f^{-1}(B) \subseteq \operatorname{ssint}\left(f^{-1}(\operatorname{int}(\operatorname{cl}(B, r, s), r, s)), r, s\right) .
$$

(5) For each fuzzy $(r, s)$-semiopen set $B$ in $Y$,

$$
\operatorname{sscl}\left(f^{-1}(B), r, s\right) \subseteq f^{-1}(\operatorname{cl}(B, r, s)) .
$$

(6) For each fuzzy $(r, s)$-semiclosed set $B$ in $Y$,

$$
f^{-1}(\operatorname{int}(B, r, s)) \subseteq \operatorname{ssint}\left(f^{-1}(B), r, s\right) .
$$

Proof. It is clear that (1) $\Leftrightarrow(2),(3) \Leftrightarrow(4),(5) \Leftrightarrow(6)$.

(2) $\Rightarrow$ (3) Let $B$ be a fuzzy $(r, s)$-closed set in $Y$. By Theorem 2.4, $\operatorname{cl}(\operatorname{int}(B, r, s), r, s)$ is fuzzy $(r, s)$-regular closed in $Y$. By $(2), f^{-1}(\operatorname{cl}(\operatorname{int}(B, r, s), r, s))$ is a fuzzy strongly $(r, s)$-semiclosed set in $X$. Hence $\operatorname{sscl}\left(f^{-1}(\operatorname{cl}(\operatorname{int}(B, r, s), r, s)), r, s\right)$

$$
\begin{aligned}
& =f^{-1}(\operatorname{cl}(\operatorname{int}(B, r, s), r, s)) \\
& \subseteq f^{-1}(\operatorname{cl}(B, r, s))=f^{-1}(B) .
\end{aligned}
$$

(3) $\Rightarrow(5)$ Let $B$ be a fuzzy $(r, s)$-semiopen set in $Y$. Then $\operatorname{cl}(B, r, s)$ is fuzzy $(r, s)$-closed in $Y$. By (3), we have $\operatorname{sscl}\left(f^{-1}(B), r, s\right)$

$$
\begin{aligned}
& \subseteq \operatorname{sscl}\left(f^{-1}(\operatorname{cl}(\operatorname{int}(B, r, s), r, s)), r, s\right) \\
& \subseteq \operatorname{sscl}\left(f^{-1}(\operatorname{cl}(\operatorname{int}(\operatorname{cl}(B, r, s), r, s), r, s)), r, s\right) \\
& \subseteq \quad f^{-1}(\operatorname{cl}(B, r, s)) .
\end{aligned}
$$

$(5) \Rightarrow(2)$ Let $B$ be a fuzzy $(r, s)$-regular closed set in $Y$. Then $B$ is fuzzy $(r, s)$-closed and fuzzy $(r, s)$-semiopen in $Y$. By (5), we obtain

$$
\begin{aligned}
f^{-1}(B) \subseteq \operatorname{sscl}\left(f^{-1}(B), r, s\right) & \subseteq f^{-1}(\operatorname{cl}(B, r, s)) \\
& =f^{-1}(B) .
\end{aligned}
$$

Thus we have $f^{-1}(B)=\operatorname{sscl}\left(f^{-1}(B), r, s\right)$, which is a fuzzy strongly $(r, s)$-semiclosed set in $X$.

Definition 3.8. Let $x_{(\alpha, \beta)}$ be an intuitionistic fuzzy point in a $\operatorname{SoIFTS}\left(X, \mathcal{T}_{1}, \mathcal{T}_{2}\right)$ and $(r, s) \in I \otimes I$. Then an intuitionistic fuzzy set $A$ in $X$ is called a fuzzy strongly $(r, s)$ semineighborhood of $x_{(\alpha, \beta)}$ if there is a fuzzy strongly $(r, s)$-semiopen set $B$ in $X$ such that $x_{(\alpha, \beta)} \in B \subseteq A$.
Theorem 3.9. Let $f:\left(X, \mathcal{T}_{1}, \mathcal{T}_{2}\right) \rightarrow\left(Y, \mathcal{U}_{1}, \mathcal{U}_{2}\right)$ be a mapping from a SoIFTS $X$ to a SoIFTS $Y$ and $(r, s) \in I \otimes I$. Then $f$ is fuzzy almost strongly $(r, s)$-semicontinuous if and only if for each intuitionistic fuzzy point $x_{(\alpha, \beta)}$ in $X$ and each fuzzy $(r, s)$-neighborhood $B$ of $f\left(x_{(\alpha, \beta)}\right)$, there is a fuzzy strongly $(r, s)$-semineighborhood $A$ of $x_{(\alpha, \beta)}$ such that $x_{(\alpha, \beta)} \in A$ and $f(A) \subseteq \operatorname{int}(\operatorname{cl}(B, r, s), r, s)$.

Proof. Let $x_{(\alpha, \beta)}$ be an intuitionistic fuzzy point in $X$ and $B$ a fuzzy $(r, s)$-neighborhood of $f\left(x_{(\alpha, \beta)}\right)$. Then there is a fuzzy $(r, s)$-open set $C$ in $Y$ such that $f\left(x_{(\alpha, \beta)}\right) \in C \subseteq B$. Thus $x_{(\alpha, \beta)} \in f^{-1}(C) \subseteq f^{-1}(B)$. Since $f$ is fuzzy almost strongly $(r, s)$-semicontinuous, by Theorem 3.7, we have

$$
\begin{aligned}
f^{-1}(C) & \subseteq \operatorname{sint}\left(f^{-1}(\operatorname{int}(\operatorname{cl}(C, r, s), r, s)), r, s\right) \\
& \subseteq \operatorname{sint}\left(f^{-1}(\operatorname{int}(\operatorname{cl}(B, r, s), r, s)), r, s\right) .
\end{aligned}
$$

Put $A=f^{-1}(\operatorname{int}(\operatorname{cl}(B, r, s), r, s))$. By Theorem 2.4, $\operatorname{int}(\operatorname{cl}(B, r, s), r, s)$ is fuzzy $(r, s)$-regular open in $Y$, and hence $A=f^{-1}(\operatorname{int}(\operatorname{cl}(B, r, s), r, s))$ is a fuzzy strongly $(r, s)$-semiopen set in $X$. Thus

$x_{(\alpha, \beta)} \in f^{-1}(C)$

$$
\begin{aligned}
& \subseteq \operatorname{ssint}\left(f^{-1}(\operatorname{int}(\operatorname{cl}(B, r, s), r, s)), r, s\right) \\
& =\operatorname{ssint}(A, r, s)=A .
\end{aligned}
$$

Hence we conclude that $A$ is a fuzzy strongly $(r, s)$ semineighborhood of $x_{(\alpha, \beta)}$ and

$$
\begin{aligned}
f(A) & \subseteq f\left(f^{-1}(\operatorname{int}(\operatorname{cl}(B, r, s), r, s))\right) \\
& \subseteq \operatorname{int}(\operatorname{cl}(B, r, s), r, s)
\end{aligned}
$$

Conversely, let $B$ be a fuzzy $(r, s)$-regular open set in $Y$ and $x_{(\alpha, \beta)} \in f^{-1}(B)$. Then $B$ is fuzzy $(r, s)$-open in $Y$, and hence $B$ is a fuzzy $(r, s)$-neighborhood of $f\left(x_{(\alpha, \beta)}\right)$. From the assumption, there is a fuzzy strongly $(r, s)$ semineighborhood $A_{x_{(\alpha, \beta)}}$ of $x_{(\alpha, \beta)}$ such that $x_{(\alpha, \beta)} \in$ $A_{x_{(\alpha, \beta)}}$ and

$$
f\left(A_{\left.x_{(\alpha, \beta)}\right)}\right) \operatorname{int}(\operatorname{cl}(B, r, s), r, s)=B .
$$

Because $A_{x_{(\alpha, \beta)}}$ is a fuzzy strongly $(r, s)$ semineighborhood of $x_{(\alpha, \beta)}$, there is a fuzzy strongly $(r, s)$-semiopen set $C_{x_{(\alpha, \beta)}}$ such that

$$
\begin{aligned}
x_{(\alpha, \beta)} \in C_{x_{(\alpha, \beta)}} & \subseteq A_{x_{(\alpha, \beta)}} \\
& \subseteq f^{-1}\left(f\left(A_{\left.x_{(\alpha, \beta)}\right)}\right) \subseteq f^{-1}(B) .\right.
\end{aligned}
$$

Hence $f^{-1}(B)=\left\{C_{x_{(\alpha, \beta)}} \mid x_{(\alpha, \beta)} \in f^{-1}(B)\right\}$, which is a fuzzy strongly $(r, s)$-semiopen set in $X$. Therefore $f$ is fuzzy almost strongly $(r, s)$-semicontinuous. 


\section{References}

[1] L. A. Zadeh, "Fuzzy sets," Information and Control, vol. 8, pp. 338-353, 1965.

[2] C. L. Chang, "Fuzzy topological spaces," J. Math. Anal. Appl., vol. 24, pp. 182-190, 1968.

[3] A. P. Šostak, "On a fuzzy topological structure," Suppl. Rend. Circ. Matem. Janos Palermo, Sr. II, vol. 11, pp. 89-103, 1985.

[4] K. C. Chattopadhyay, R. N. Hazra, and S. K. Samanta, "Gradation of openness : Fuzzy topology," Fuzzy Sets and Systems, vol. 49, pp. 237-242, 1992.

[5] A. A. Ramadan, "Smooth topological spaces," Fuzzy Sets and Systems, vol. 48, pp. 371-375, 1992.

[6] K. T. Atanassov, "Intuitionistic fuzzy sets," Fuzzy Sets and Systems, vol. 20, pp. 87-96, 1986.

[7] D. Çoker, "An introduction to intuitionistic fuzzy topological spaces," Fuzzy Sets and Systems, vol. 88, pp. 81-89, 1997.

[8] D. Çoker and M. Demirci, "An introduction to intuitionistic fuzzy topological spaces in Sostak's sense," BUSEFAL, vol. 67, pp. 67-76, 1996.

[9] S. Z. Bai, "Fuzzy almost strong semicontinuous mappings," Bulletin for Studies and Exchanges on Fuzziness and its Applications, vol. 62, pp. 89-93, 1995.
[10] E. P. Lee, "Semiopen sets on intuitionistic fuzzy topological spaces in Sostak's sense," J. Fuzzy Logic and Intelligent Systems, vol. 14, pp. 234-238, 2004.

[11] S. J. Lee and J. T. Kim, "Fuzzy $(r, s)$-irresolute maps," International Journal of Fuzzy Logic and Intelligent Systems, vol. 7, no. 1, pp. 49-57, 2007.

[12] S. J. Lee and J. T. Kim, "Fuzzy almost $(r, s)$ semicontinuous mappings," Commun. Math. Math. Sci., vol. 6, pp. 1-9, 2010.

[13] S. O. Lee and E. P. Lee, "Fuzzy strongly $(r, s)$ semiopen sets," International Journal of Fuzzy Logic and Intelligent Systems, vol. 6, no. 4, pp. 299-303, 2006.

[14] E. P. Lee and S. H. Kim, "Fuzzy strongly $(r, s)$ semicontinuous, fuzzy strongly $(r, s)$-semiopen, fuzzy strongly $(r, s)$-semiclosed mappings," International Journal of Fuzzy Logic and Intelligent Systems, vol. 7, no. 2, pp. 120-126, 2007.

\section{Seok Jong Lee}

Professor of Chungbuk National University

Research Area: Fuzzy mathematics, Fuzzy topology, General topology

E-mail : sjl@chungbuk.ac.kr

\section{Jin Tae Kim}

Research Area: Fuzzy mathematics, Fuzzy topology, General topology

E-mail : kjtmath@hanmail.net 